\title{
Le dispositif des sections européennes
}

Yvonne Pernet

\section{(2) OpenEdition}

\section{Journals}

Édition électronique

URL : http://journals.openedition.org/ries/3962

DOI : 10.4000/ries.3962

ISSN : 2261-4265

\section{Éditeur}

Centre international d'études pédagogiques

\section{Édition imprimée}

Date de publication : 7 septembre 1995

Pagination : 79-85

ISSN : 1254-4590

\section{Référence électronique}

Yvonne Pernet, «Le dispositif des sections européennes », Revue internationale d'éducation de Sèvres

[En ligne], 07 | 1995, mis en ligne le, consulté le 14 novembre 2019. URL : http://

journals.openedition.org/ries/3962 ; DOI : 10.4000/ries.3962

Ce document a été généré automatiquement le 14 novembre 2019.

(c) Tous droits réservés 


\title{
Le dispositif des sections européennes
}

\author{
Yvonne Pernet
}

1 Les sections européennes ont été créées à la rentrée $1992^{1}$. L'objectif vise, par un temps d'exposition plus long à la langue, à développer chez les élèves les compétences linguistiques et à leur faire acquérir une connaissance approfondie du pays dont ils apprennent la langue. L'ouverture d'une telle section est décidée par le recteur, sur proposition des établissements, après avis d'une commission composée d'inspecteurs d'académie, d'inspecteurs pédagogiques régionaux de langues et de disciplines non linguistiques, de chefs d'établissement.

2 Le dispositif prévoit pendant les deux premières années un horaire renforcé de la langue, à raison d'au moins deux heures hebdomadaires en plus de l'horaire normal.

3 La section européenne commence en classe de quatrième; elle peut débuter exceptionnellement en sixième afin d'assurer un suivi aux élèves ayant bénéficié d'un enseignement de langue à l'école primaire.

4 Après ces deux années d'enseignement renforcé, une discipline non linguistique sera enseignée "pour tout ou partie» dans la langue considérée ${ }^{2}$. Il semble toutefois raisonnable de n'enseigner qu'une partie du programme. Une note de service ${ }^{3}$ va dans ce sens puisqu'il y est fait mention d'un « enseignement partiel d'une discipline non linguistique dans la langue de la section ». Dans cette deuxième phase, la langue étrangère n'est plus objet d'étude mais devient langue de communication.

5 La langue de la section européenne est, en général, une langue européenne. Mais il est possible d'ouvrir une section avec d'autres langues, à la demande des familles. Ainsi existe-t-il quelques sections en arabe, en chinois, en japonais. Elles sont alors appelées « sections de langues orientales».

6 Le choix de la langue est fait en fonction de ressources de l'établissement, des compétences linguistiques des enseignants de la discipline non linguistique ou des possibilités d'intervention extérieure. La langue vivante peut être la langue 1 ou la langue 2. En allemand et en anglais, c'est principalement la langue 1 qui est concernée. 
7 Le fonctionnement est en règle générale financé par la dotation globale des établissements. Tout établissement secondaire - général, technique, professionnel - est autorisé à ouvrir une section européenne si les conditions préalables à l'ouverture sont respectées. Cependant peu de lycées techniques et encore moins de lycées professionnels proposent une section européenne à l'exception des académies de l'Est pour l'allemand. Un tel projet doit s'intégrer dans un projet culturel international et s'associer à des établissements étrangers.

8 Un dispositif semblable existe dans d'autres pays, avec des appellations différentes (classes bilingues en République fédérale d'Allemagne ou dans les pays d'Europe centrale) mais avec un objectif commun : l'acquisition progressive d'un bilinguisme plus ou moins développé.

9 Précisons toutefois que dans des pays comme l'Allemagne, la République Tchèque, la Slovaquie, la Hongrie, pour ne citer que quelques exemples, la durée d'apprentissage de la discipline non linguistique en langue étrangère est plus longue: sept ans en Allemagne, quatre ou cinq ans en République tchèque ou en Slovaquie.

\section{Implantation}

10 Il est difficile de faire avec précision un état des lieux. L'enthousiasme des débuts peut ne pas se poursuivre; il faut toujours compter avec une certaine érosion. Par ailleurs la notion de « sites » n'a pas été, dans un premier temps du moins, suffisamment prise en considération. Un grand nombre de collèges ont ouvert une section européenne, sans s'assurer de la continuité en aval. La mise en place d'une organisation cohérente, la nécessaire articulation entre les collèges et le lycée d'accueil sont les principes essentiels à respecter avant tout projet d'ouverture.

11 Dès l'annonce de la création, à la rentrée 1992, cent cinq sections européennes s'ouvraient dans quinze académies (cinquante-six en anglais, trente-deux en allemand, neuf en espagnol, six en italien, deux en portugais).

12 À la rentrée 1993, deux cents nouvelles sections européennes se créent dans presque toutes les académies. On voit apparaitre des langues moins enseignées telles le russe avec trois sections, l'arabe avec une section.

13 Actuellement on dénombre six-cent-dix-sept sections européennes en collège - avec apparition du chinois et du japonais et cent-cinquante-deux en lycée : soixante-huit en anglais, soixante-trois en allemand, douze en espagnol, huit en italien, une en arabe.

14 Le nombre de sections européennes ne dépend ni de la taille ni de la population des académies; ainsi l'académie de Créteil a dix sections européennes en collège et une seule en lycée tandis que dans l'académie d'Amiens leur nombre s'élève respectivement à dix et une et dans celle de Limoges à neuf et quatre.

Certaines académies sont très engagées dans cette politique internationale. L'académie de Strasbourg avec ses quatre-vingt-dix sections en collège et ses quarante-quatre en lycée est particulièrement bien représentée. La proximité de l'Allemagne n'entrave nullement la diversité linguistique puisque l'on compte vingt-cinq lycées avec des sections en allemand, quinze lycées avec des sections en anglais, deux lycées avec des sections en italien, un lycée avec une section en espagnol, un lycée avec une section en arabe. 


\section{Recrutement des élèves} d'établissement s'efforcent de regrouper les élèves de la section européenne au sein d'une même division, ce qui rend la gestion administrative et pédagogique plus aisée. Dans le cas contraire, les élèves de cette section suivent le cours de langue avec un autre groupe pendant les trois heures réglementaires et travaillent de façon autonome pendant les deux heures de renforcement linguistique, ce qui ne manque pas de poser un problème pour la mise en œuvre d'un projet spécifique à cette section.

\section{La discipline non linguistique}

Des constatations s'imposent. Des questions se posent.

\section{Quelle discipline non linguistique?}

C'est l'histoire et la géographie qui sont proposées à une très large majorité, pour plusieurs raisons. Ces disciplines favorisent la communication orale et les prises de position personnelles, donc l'expression; elles sont propices à une réflexion sur le sens exact des mots; elles permettent d'intégrer une démarche interculturelle, une dimension européenne, voire internationale; ce sont les seules matières dont le programme soit commun à l'ensemble des filières. 
D'autres disciplines sont également proposées, mais à un degré moindre : la biologie, l'éducation physique et sportive, la physique-chimie, les mathématiques et, dans les lycées techniques et professionnels, l'hôtellerie, la restauration, la vente.

En règle générale, une à deux heures hebdomadaires sont consacrées à la discipline non linguistique. Cet horaire devrait intervenir en sus de l'horaire normal et non en substitution.

Des questions restent posées : peut-on changer de discipline non linguistique en cours de cycle? Peut-on choisir plusieurs disciplines non linguistiques dans une même année?

Dans l'état actuel, il est difficile de trouver une réponse précise, même si des exemples à l'étranger semblent fournir une solution, dans un contexte certes différent.

Si l'ouverture à un vocabulaire spécifique et diversifié, si l'utilisation de la langue étrangère dans des situations variées facilitent une mobilisation plus intense des acquis linguistiques, on peut craindre en revanche une surcharge de travail pour les élèves, un risque d'éparpillement et un manque d'approfondissement. Se poserait par ailleurs la difficulté d'une évaluation au baccalauréat.

\section{Quels professeurs?}

Seuls les maitres habilités à enseigner la discipline non linguistique et possédant des connaissances suffisantes en langues peuvent prétendre occuper ce poste. Leur compétence doit être triple : scientifique, linguistique, pédagogique.

Or les professeurs en France n'étant pas bivalents, il est souvent difficile de promouvoir une section européenne faute d'enseignants aptes à dispenser leur enseignement en langue étrangère.

31 Dans beaucoup de cas cependant, l'enseignement de la discipline non linguistique est assurée par le professeur de la classe, spécialiste de la discipline et possédant une compétence reconnue en langue, attestée par 1' inspecteur pédagogique régionalinspecteur d'académie (IPR-IA) de cette langue.

D'autres possibilités peuvent être envisagées : échange de professeurs venant d'autres établissements, échange "poste pour poste ", interventions ponctuelles de collègues étrangers (se pose alors le problème de leur statut et de leur rémunération).

Il conviendra de prévoir des stages de mise à niveau et de formation continue- ce qui se fait d'ailleurs déjà çà et là.

En effet, l'enseignement bilingue doit s'apprendre. Il ne suffit pas de parler la langue étrangère. Il faut, d'une part, connaître le système éducatif du pays partenaire et transmettre, d'autre part, des savoirs dans la langue étrangère.

Aussi, les Instituts universitaires de formation des maitres (IUFM) pourraient-ils jouer un rôle dans cette formation spécifique. Elle existe déjà à 1'ITUFM de Strasbourg qui propose au cours de la seconde année une formation de vingt jours en allemand. L'accent est mis sur le système éducatif en Allemagne, les programmes, la pédagogie de la discipline. Un stage de dix jours est effectué auprès d'un professeur allemand, avec en conclusion la rédaction d'un mémoire. Ce stage se termine par un séjour culturel et linguistique en Allemagne et la remise officielle d'une attestation. 


\section{Quelle pédagogie?} maîtrise de la langue. L'objectif disciplinaire est prépondérant. Un cours d'histoire, de géographie, de biologie n'est pas un cours de langue. Le professeur de discipline non linguistique ne doit pas se substituer au professeur de langue.

Cette finalité est parfois mal interprétée. La limite entre le renforcement linguistique et l'enseignement de la discipline non linguistique n'est - pour l'instant du moins - pas toujours bien marquée. L'étude de la civilisation - partie intégrante de tout enseignement linguistique - est souvent seule prise en compte. Si elle s'insère naturellement dans son cadre, en histoire-géographie particulièrement, elle ne doit pas se limiter à cet aspect.

C'est un enseignement concret, centré sur l'élève que le professeur de la discipline non linguistique se doit de dispenser, en évitant le flou artistique et le vagabondage linguistique. La discipline doit garder sa rigueur. La progression sera nécessairement plus lente au début. Mais la section européenne s'adresse en principe à des élèves motivés; il sera ainsi vite remédié à ce ralentissement. On peut se demander, par ailleurs, si ce rythme plus lent ne favorisera pas la conceptualisation de certaines notions et un meilleur ancrage des connaissances.

\section{Quel programme?}

La question se pose de savoir si toutes les parties du programme peuvent être enseignées en langue étrangère et si la composante culturelle ne risque pas de l'emporter sur les connaissances spécifiques. En tout état de cause, il serait judicieux de sélectionner certains thèmes plus faciles à traiter en langue étrangère.

40 Si en classe de seconde et en classe de première le choix peut sembler relativement aisé, en classe de terminale il sera plus difficile de concilier les objectifs de cette section et de l'examen final.

\section{Quels outils pédagogiques?}

41 Disons-le d'emblée : ils n'existent pas encore. Le professeur doit constituer lui-même sa documentation, faire des recherches personnelles et enrichir sans cesse ses connaissances d'apports nouveaux.

Certains manuels étrangers peuvent être proposés aux élèves : ils devront toutefois être utilisés avec précaution, l'approche linguistique étant trop difficile et les programmes ne correspondant pas nécessairement aux nôtres. Il ne convient cependant pas de les rejeter, mais il faut en conseiller de temps en temps l'usage afin que les élèves découvrent d'autres horizons, d'autres méthodes pédagogiques.

Une collaboration entre spécialistes de pays partenaires pourrait contribuer à l'élaboration d'un matériel didactique. 


\section{Certification}

Les premiers candidats se présenteront à la session 1995 pour l'obtention de la " mention spéciale européenne ${ }^{4}$ ». Ils passeront l'épreuve normale de langue et devront obtenir une note équivalente à, au moins, 14 sur 20. Ils auront, en outre, une note supplémentaire, calculée à raison de $20 \%$ sur le travail fourni en discipline non linguistique au cours de la classe de terminale et de $80 \%$ après épreuve orale spécifique passée sur présentation de dossiers devant un jury composé d'examinateurs de langue et de la discipline concernée. Cette note devra être supérieure à 10 sur 20.

\section{Conditions de réussite et perspectives d'avenir}

Les apports de la section européenne sont indéniables à maints égards sur le plan pédagogique (meilleure relation entre professeurs et élèves; exigence d'une plus grande rigueur; décloisonnement entre les disciplines; réflexion pédagogique renouvelée ; ouverture sur l'Europe et le monde, etc.).

La réussite n'en sera assurée que si l'on tient compte de certains principes et éléments concernant l'intégration de la section européenne dans le projet d'établissement; l'élaboration d'un projet commun entre les collèges et le lycée d'accueil ; la création dans chaque académie d'un groupe de pilotage où l'IPR-IA de langue et l'IPR-IA de la discipline non linguistique réfléchiront et travailleront ensemble ; l'implication du chef d'établissement (intégration de la composante européenne dans le projet d'établissement, jumelage avec des lycées bilingues à l'étranger, développement des séjours scolaires à l'étranger avec une dimension pédagogique précise et rigoureuse, installation d'un équipement moderne et adapté au niveau du centre de documentation et d'information...); le développement des stages linguistiques à l'intention des professeurs de la discipline non linguistique ; l'étude renforcée d'une langue dans les instituts universitaires de formation des maitres à l'intention des stagiaires non linguistes; la concertation entre professeurs de langue et professeurs de disciplines non linguistiques.

Les sections européennes ont déjà prouvé leur dynamisme et leur efficacité. Pour qu'elles se réalisent pleinement, il conviendrait, dans un premier temps du moins, de privilégier la qualité à la quantité.

\section{NOTES}

1. Circulaire n 92.234 du 19 août 1992.

2. BO $n^{\circ} 33$ du 3 septembre 1992.

3. BO $n^{\circ} 15$ du 13 avril 1995.

4. B0 $\mathrm{n}^{\circ} 2$ du 21 juillet 1994. 
INDEX

Index géographique : France

Mots-clés : enseignement bilingue, formation des enseignants, section européenne, section bilingue, discipline non linguistique

\section{AUTEUR}

\section{YVONNE PERNET}

Doyen de l'Inspection générale d'allemand, Paris, France 